\title{
A UNIT-CELL APPROACH TO PREDICTION OF THE MICROBUCKLING STRENGTH OF COMPOSITES WITH NON-UNIFORM FIBER IMPERFECTIONS UNDER COMBINED AXIAL COMPRESSION AND IN-PLANE SHEAR
}

\author{
Marek Romanowicz \\ Bialystok University of Technology, Department of Mechanical Engineering, Bialystok, Poland \\ e-mail:m.romanowicz@pb.edu.pl
}

\begin{abstract}
A new micromechanical model for predicting the failure locus of long fiber composites under combined axial compression and in-plane shear is proposed. The model is based on a periodic unit cell with centrally located imperfections. Predictions of the compressive behavior for various biaxial loading ratios are made. The role of distribution of fiber imperfections in predicting the biaxial strength is discussed. The failure locus calculated from the new model is found to be in good agreement with experimental data available in the literature and less conservative than that from the periodic model with uniform imperfections.
\end{abstract}

Keywords: fiber-reinforced composites, buckling, micro-mechanics, finite element analysis

\section{Introduction}

Experimental observations have shown that damage in long fiber composites due to axial compression localizes into bands of highly bent fibers with distinct inclinations and widths, whose normals lie in one plane (Kyriakides et al., 1995; Kyriakides and Ruff, 1997). This planar behavior of kink bands explains why simplified two-dimensional finite element representations of the composite microstructure with a ductile matrix are commonly used to reproduce the compression behavior. In the literature, there is a large variety of finite element models for analyzing the plastic microbuckling. Although, the models differ in the constitutive equations employed by authors to describe the behavior of composite constituents, the fiber waviness is typically idealized with uniform imperfections. In general, two types of boundary conditions are enforced in numerical studies of the compressive failure. In the first case, the longitudinal sides of the model are free from constraints. The model with free boundary conditions is typically composed of several dozen alternate layers of the fibers and matrix explicitly modeled by finite elements. It has been used extensively by various research groups: see, for example, Kyriakides et al. (1995), Kyriakides and Ruff (1997), Hsu et al. (1998), Vogler et al. (2001), Byskov et al. (2002), Pimenta et al. (2009) and Prabhakar and Waas (2013). In the second case, periodic boundary conditions are prescribed on the longitudinal sides of the model. The model with periodic boundary conditions usually consists of one fiber embedded in the matrix. The concept of the periodic unit cell model has been employed for studying compressive failure in unidirectional composites in papers by Guynn et al. (1992), Morais (1996), Hsu et al. (1999), Pansart et al. (2009), Gutkin et al. (2010a), Barulich et al. (2016), Naya et al. (2017). Both types of boundary conditions have advantages and limitations. On the one hand, the periodic boundary conditions force deformation to be symmetric regardless of the geometry of the fiber waviness. This means that the non-periodic model with a uniform waviness can ensure a realistic inclination of kink bands, contrary to the periodic model with uniform waviness in which the kink-band angle is indirectly set to zero degree. On the other hand, the free boundary conditions produce 
severe stress concentrations at the free surfaces which can lower the buckling stress. In order to overcome these limitations, Romanowicz (2014) proposed a new approach based on the periodic model with centrally located imperfections. This approach is motivated by experimental observations (Kyriakides et al., 1995; Kyriakides and Ruff, 1997; Czabaj et al., 2014) that fiber imperfections are more severe in matrix-rich regions which are periodically distributed along the transverse direction. A comparison of the new model with those reported in the literature on the compressive failure showed that it both calculates the limit load well and, in addition, predicts the post-critical events correctly. Recently, Wilhelmsson et al. (2018) investigated the effects different fiber waviness characteristics on the compressive properties of unidirectional non-crimp fabric composites. The results of this experimental study support the importance of non-uniform imperfections in the modeling of the compression behavior.

Other experimental studies show that shear applied simultaneously with compression decreases the strength of fiber-reinforced composites (Jelf and Fleck, 1994; Vogler et al., 2000). This paper presents an extension of the periodic model with centrally located imperfections proposed by Romanowicz (2014) to the case of combined compression and shear. In order to validate the proposed model, a comparison with both the existing models and experimental results will be presented. To date, the failure loci for the case of combined compression and shear have been determined numerically from the classical approaches with uniform imperfections (Gutkin et al., 2010b, 2011; Vogler et al., 2000). According to the classical models, there is no change in the failure locus when the failure mode changes from fiber kinking to fiber splitting. The simulations with non-uniform imperfections offer some important insights into the role played by the distribution of imperfections in controlling the biaxial strength.

\section{Description of the micromechanical model}

\subsection{Microstructure generation}

A unidirectional composite is idealized as a two-dimensional periodic array of imperfect fibers and a matrix. Following Kyriakides et al. (1995) and Kyriakides and Ruff (1997), it is assumed that the fiber imperfection is not uniformly distributed across the model. The imperfection shown in Fig. 1a consists of a sinusoidal waviness of the fibers along the $x_{1}$ direction with a variable amplitude in the $x_{2}$ direction. The amplitude of the imperfection has its maximum at the center of the model and decays exponentially with the distance from the mid-width, as follows

$$
\nu_{o}=A \cos \frac{\pi x_{1}}{\lambda} \exp \left[-\zeta\left(\frac{2 x_{2}}{\alpha b}\right)^{2}\right]
$$

where $A$ is the amplitude, $\lambda$ is the half-wavelength, $b$ is the width of the model, $\alpha$ is the ratio of the number of imperfect fibers to that of the total number of fibers in the model, $\zeta$ defines how fast the amplitude diminishes. The models with non-uniform distributions of imperfections characterized by $\zeta=\ln (0.01)$ and $\alpha=0.50,0.76$ and 1.00 are considered. The spacing of the fibers is chosen so as to accommodate the fiber diameter $d_{f}=7 \mu \mathrm{m}$, and to respect the fiber volume content $V_{f}=60 \%$. The models have width $b$ defined by the number of fibers $n_{f}=59$. For a long wavelength and a small amplitude, the fiber misalignment angle can be approximated by

$$
\Theta=\frac{\pi A}{\lambda}
$$

Base case calculations are performed for three amplitudes $A=2 d_{f}, 3 d_{f}, 4 d_{f}$ and $5 d_{f}$ and one fixed length of the models $L=75 d_{f}$ corresponding to the half-wavelength $\lambda=L$. This 
(a)

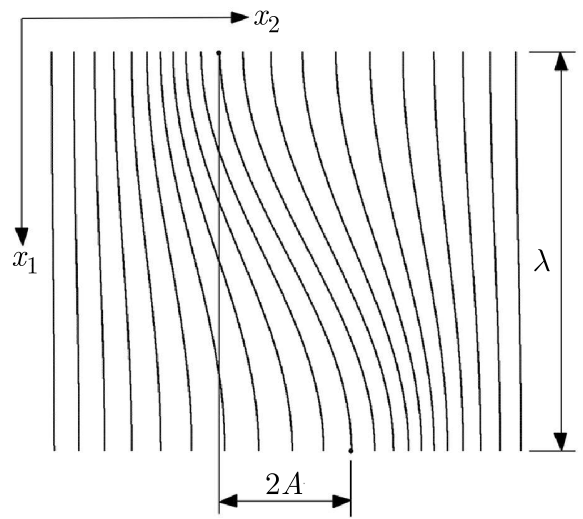

(b)
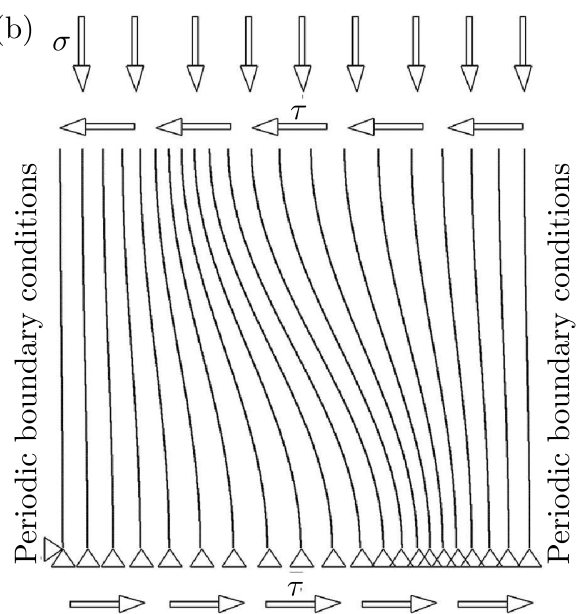

Fig. 1. Micromechanical model with a non-uniform distribution of fiber imperfections: (a) geometry, (b) boundary conditions

implies the maximum values of fiber misalignment angle $\Theta_{\max }$ at the centers of the models of approximately $4.80^{\circ}, 7.20^{\circ}, 9.60^{\circ}$ and $12^{\circ}$. Please note, that if $\zeta=0$, Eq. (2.1) describes a fiber imperfection which is uniformly distributed across the model. In this case, the amplitude of the imperfection is fixed in the $x_{2}$ direction. The models with uniform distributions of imperfections characterized by $\zeta=0$ and $\Theta=1.03^{\circ}, 1.71^{\circ}, 2.40^{\circ}$ and $3.42^{\circ}$ are considered for comparison purposes. Figure 2 illustrates the difference in the distribution of fiber imperfections between the two cases.

(a)

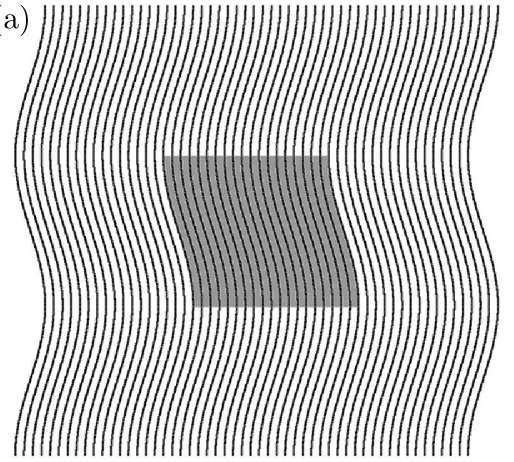

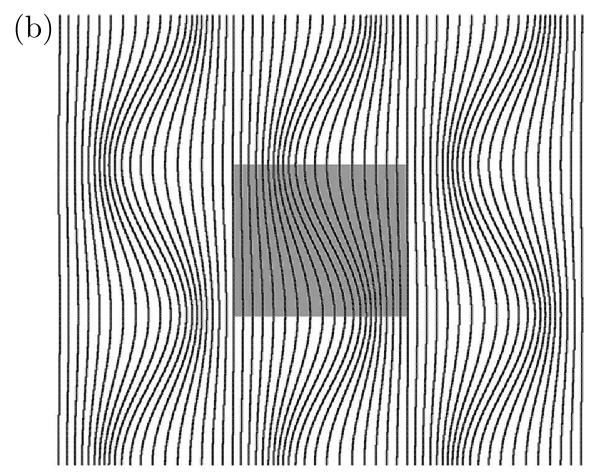

Fig. 2. Distribution of fiber imperfections: (a) uniform, (b) non-uniform

\subsection{Finite element formulation}

Two-dimensional finite element meshes made of plane strain, biquadratic elements with eight nodes (Plane183) are generated for appropriate geometries of micromechanical models by using ANSYS finite element code. The fiber and matrix layers each have 1575 and 2100 elements, respectively. The mesh refinement is found to be adequate for a sufficient solution accuracy. The boundary conditions are shown in Fig. 1b. In simulations of combined compression and shear, the shear is applied first. The shear loading is set by two opposite shear forces at the top and bottom sides so that the two sides remain plane. The model is then compressed in the vertical direction by fixing the bottom side and prescribing a compressive force at the top side. The biaxial loading ratio is defined by the relation $\kappa=\tau / \sigma$. This two-step loading process corresponds to the loading history used in biaxial testing. Periodic boundary conditions on the longitudinal sides of the model are applied to reproduce the periodicity of fiber imperfections. 
The displacements of nodes on the right side of the model are related to the displacements of their counterparts on the left side, as follows

$$
u_{i}^{n_{2}}-u_{i}^{r e f_{2}}=u_{i}^{n_{1}}-u i^{r e f_{1}} \quad \text { for } \quad i=1,2
$$

where $u_{i}$ is the displacement in the $i$-axis direction, $n_{1}$ and $n_{2}$ is a pair of nodes facing each other on edge 1 and edge 2, respectively, ref $f_{1}$ and $r e f_{2}$ is a pair of reference nodes on edge 1 and edge 2. The assumption of transverse periodicity means that the lateral edges of the model do not affect the state of stress within it. In order to simulate the post-buckling response effectively, the arc-length method (Crisfield, 1981) is used. The minimum arc-length radius is defined by the minimum multiplier of reference arc-length radius $r_{\min }=10^{-8}$ and the number of substeps $n=5000$. Convergence of the iterative procedure has been achieved by requiring that the force equilibrium is satisfied with a tolerance of $t_{0} l_{F}=0.001$.

\subsection{Constituent properties}

The material properties of the AS4/PEEK composite analyzed in this paper are listed in Table 1. The fibers are assumed to be isotropic and linearly elastic solids which are perfectly bonded to the matrix. The matrix is modeled within the framework of the finite deformations as an elasto-plastic solid which hardens isotropically.

Table 1. Mechanical properties of the AS4/PEEK composite and its constituents

\begin{tabular}{|c|c|c|c|c|c|c|c|c|c|}
\hline \multicolumn{3}{|c|}{ AS4 fiber } & \multicolumn{4}{c|}{ PEEK matrix } & \multicolumn{2}{c|}{ AS4/PEEK composite } \\
\hline $\begin{array}{c}E_{f} \\
{[\mathrm{GPa}]}\end{array}$ & $\begin{array}{c}\nu_{f} \\
{[-]}\end{array}$ & $\begin{array}{c}\sigma_{c}^{+} \\
{[\mathrm{GPa}]}\end{array}$ & $\begin{array}{c}E_{m} \\
{[\mathrm{GPa}]}\end{array}$ & $\begin{array}{c}\nu_{m} \\
{[-]}\end{array}$ & $\begin{array}{c}\mu \\
{[-]}\end{array}$ & $\begin{array}{c}k \\
{[\mathrm{MPa}]}\end{array}$ & $\begin{array}{c}E_{11} \\
{[\mathrm{GPa}]}\end{array}$ & $\begin{array}{c}\sigma_{c}^{-} \\
{[\mathrm{GPa}]}\end{array}$ & $\begin{array}{c}V_{f} \\
{[\%]}\end{array}$ \\
\hline \hline 214 & 0.263 & 3.45 & 6.14 & 0.356 & 0.1 & 40 & 128 & 1.21 & 60 \\
\hline
\end{tabular}

Due to using the Drucker-Prager plasticity model (Drucker and Prager, 1952), a linear dependency of yielding on the hydrostatic stress is taken into account in this paper. In terms of the first invariant of stress $I_{1}$ and the second invariant of the deviatoric part of stress $J_{2}$, the yield function is given as

$$
f=\frac{\mu I_{1}}{3}+\sqrt{J_{2}}-k
$$

where $\mu$ is the pressure sensitivity factor, $k$ is the flow stress of the material under pure shear. Motivated by experimental results found in the literature (Kinloch and Young, 1983; Quinson et al., 1997), a value of $\mu=0.1$ is adopted in this paper. Please note, that if $\mu=0$, Eq. (2.4) reduces to the von Mises yield function. An associative flow rule is used to characterize the plastic flow. Micromechanical models require in-situ properties of matrices that differ from bulk properties (Gregory and Spearing, 2005). Here, the hardening curve of the matrix is identified so that the unit cell prediction matches the measured in-plane shear response reported for the AS4/PEEK composite system (Vogler et al., 2000). For this purpose, a two-dimensional unit cell model with perfectly straight fibers and periodic boundary conditions is used. Figure 3 shows a comparison of the measured shear response and that obtained from the unit cell model. Agreement with the experimental data is quite good.

\section{Results and discussion}

\subsection{Failure predictions under combined axial compression and in-plane shear}

The micromechanical models must be calibrated before they are used for predicting the microbuckling strength of fiber reinforced composites under combined axial compression and 


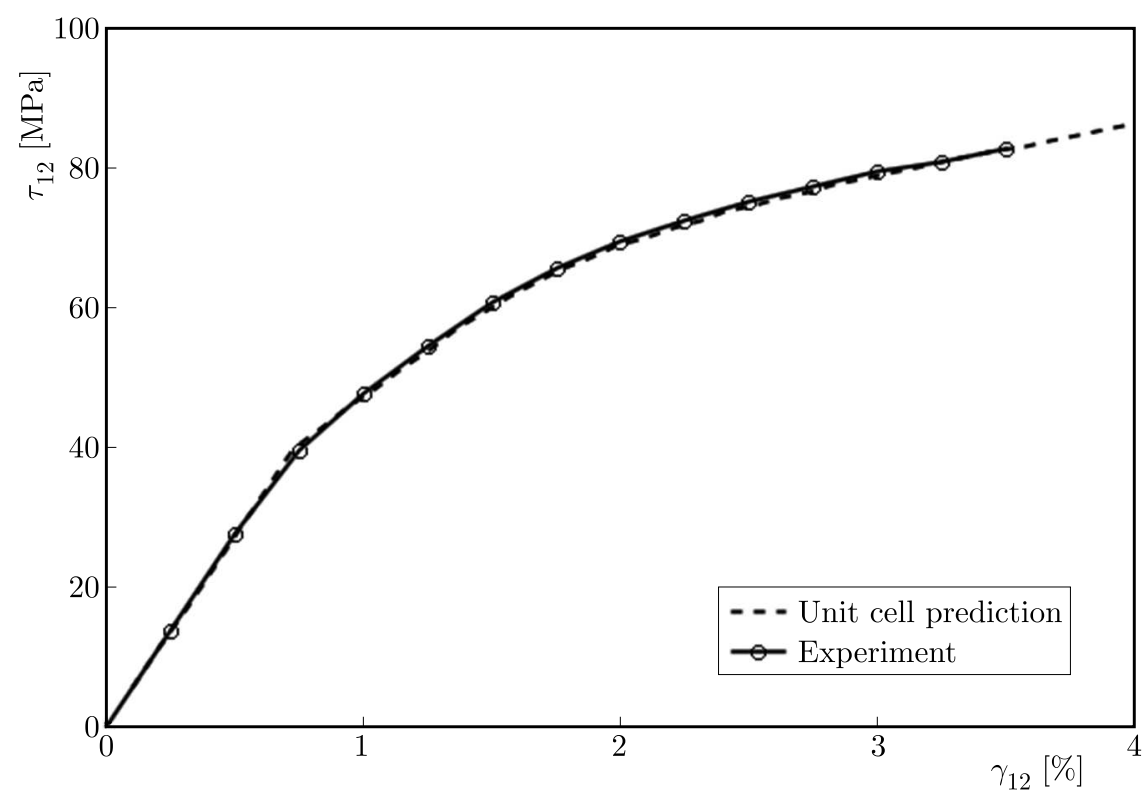

Fig. 3. Calibration of the Drucker-Prager plasticity model

in-plane shear. In order to determine imperfection characteristics, a parametric analysis under pure compressive loading is carried out for two imperfection types. The effect of imperfection parameters is discussed in Romanowicz (2014) and will not be repeated here. In the case of the AS4/PEEK composite, the model with a non-uniform distribution of imperfections characterized by $\alpha=0.76$ and $\Theta_{\max }=9.60^{\circ}$ shows good agreement with the measured compressive strength $\sigma_{c}=1.21 \mathrm{GPa}$ reported by Kyriakides et al. (1995). A similar calibration has been carried out for the uniform model. It was found that the model prediction for $\Theta=2.40^{\circ}$ matched well with the experimental strength data for that fiber reinforced composite.

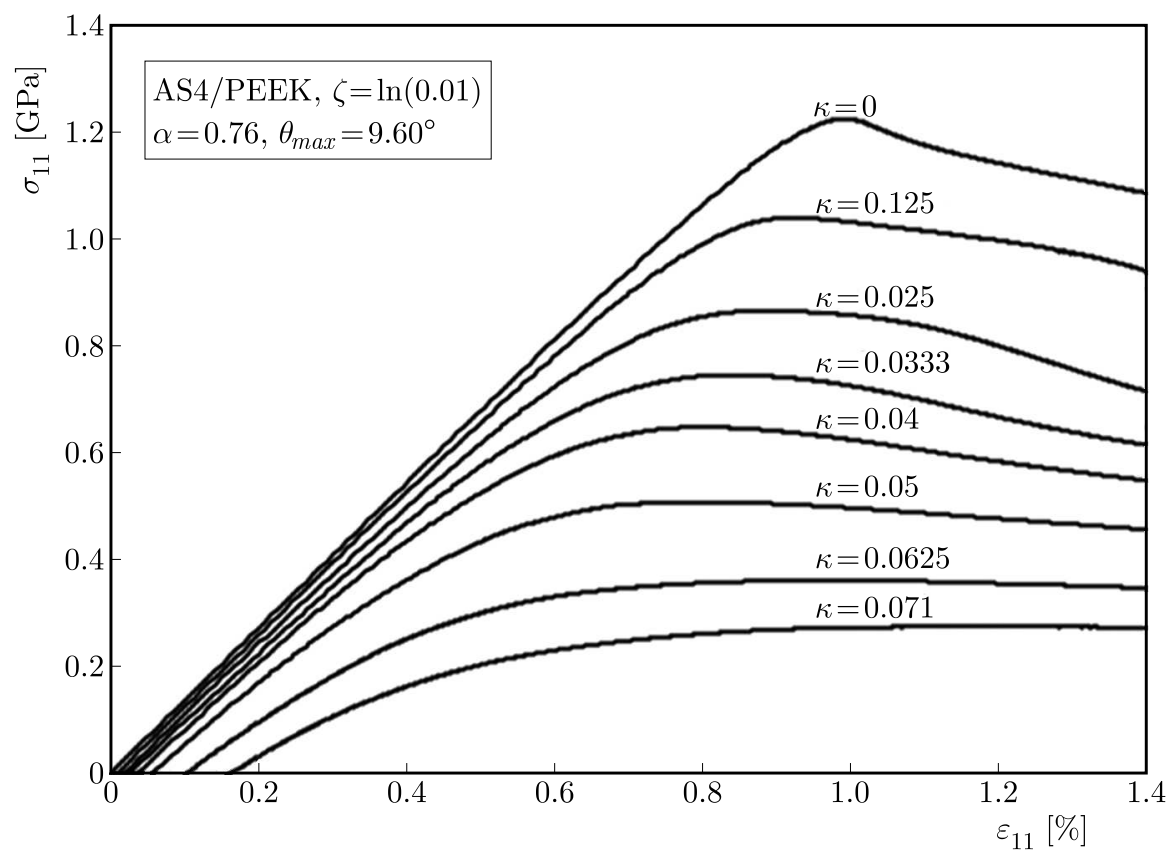

Fig. 4. Effect of the biaxial loading ratio $\kappa$ on the compressive stress-strain curve of the AS4/PEEK composite system. The solutions obtained from the model with non-uniform imperfections

Based on the above-mentioned parameters, the failure process under combined axial compression and in-plane shear is then modeled. The effect of shearing applied before axial compression 


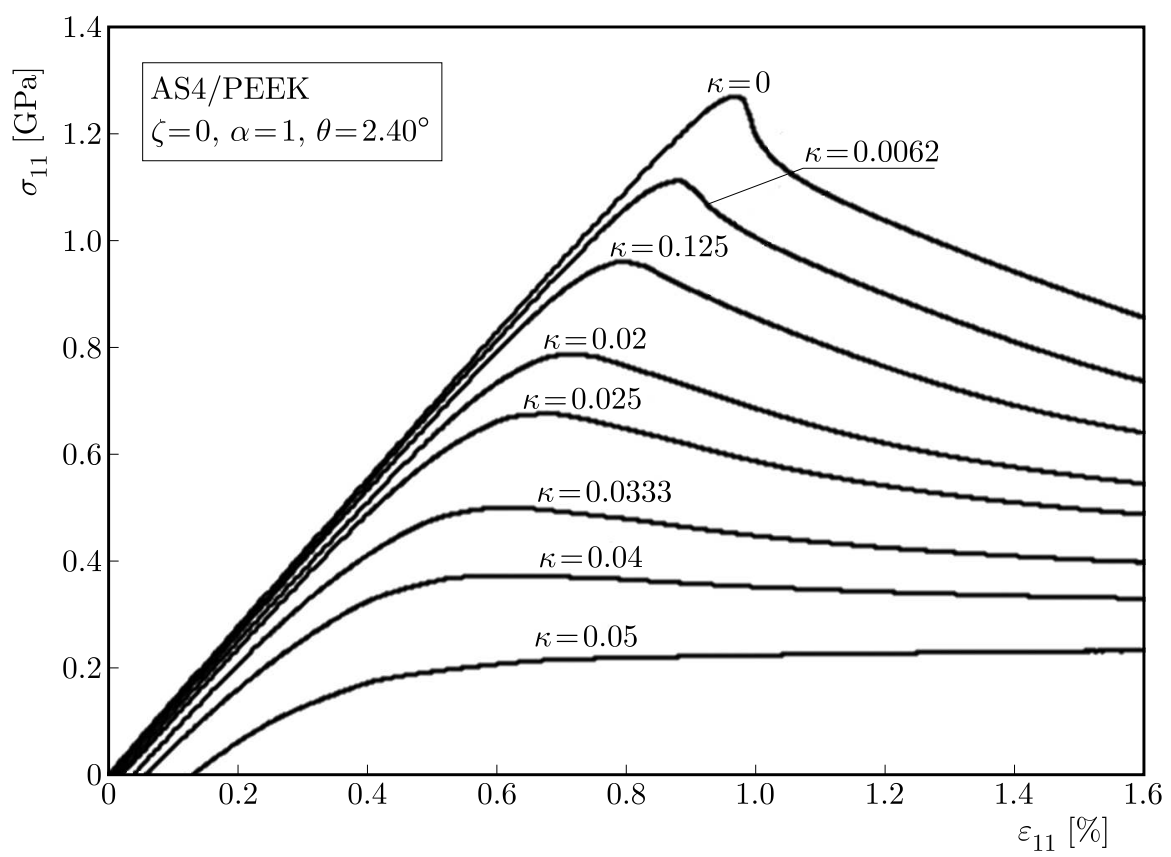

Fig. 5. Effect of the biaxial loading ratio $\kappa$ on the compressive stress-strain curve of the AS4/PEEK composite system. The solutions obtained from the model with uniform imperfections

on the compressive response is shown in Figs. 4 and 5 for two imperfection types. In each case, calculations are carried out for eight biaxial loading ratios $\kappa$ given in these figures. The compressive curves are shifted from the point of zero strain because the initial shearing produces some compressive strain. It can be seen that the presence of the initial shear loading decreases significantly the compressive strength and reduces the sharpness of the snap-through behavior after the limit load. It is interesting to note that a similar effect is observed in the case of pure compression for which the buckling instability weakens as the imperfection parameters $\alpha$ and $\Theta_{\max }$ increase (Romanowicz, 2014). Moreover, as the value of the ratio $\kappa$ increases, the limit load occurs at a lower applied strain level. For sufficiently large values of $\kappa$, the stress-strain curves exhibit no apparent instability. This finding suggests that another mode of failure is operating.

Figure 6 clearly shows that a variation of the maximum axial stress obtained from a fiber located at the center of a non-uniform model is a representative of the evolution of this stress in other fibers. The effect of the initial shear loading on the stress state in fibers for all cases considered above is shown in Figs. 7 and 8. It can be seen that shearing causes an increase in the tensile stress in the central fiber during the shear loading stage. As the value of the ratio $\kappa$ increases, the central fiber reaches the buckling instability at a lower applied strain level. For small values of $\kappa$, the axial response during the compressive loading stage is similar to the case of pure compression. This means that the axial stress in the central fiber decreases first, reaches a negative value and then starts to increase again. The minimum value of the axial stress defines the resistance to bending. It is evident from these figures that the resistance to bending of the central fiber decreases with increasing the applied shear loading. For large values of $\kappa$, the buckling instability is inhibited by the presence of high tensile stress in fibers arising from shear loading. Finally, for sufficiently large values of $\kappa$, the fiber buckling mechanism is replaced by the stable fiber bending mechanism in which the axial stress in fibers increases constantly and has no minimum value. Such behavior has not been observed in the uniform model $(\zeta=0)$ in which the minimum value exists for large values of $\kappa$. These results provide further support that unidirectional composites fail in a stable manner for large values of $\kappa$. 


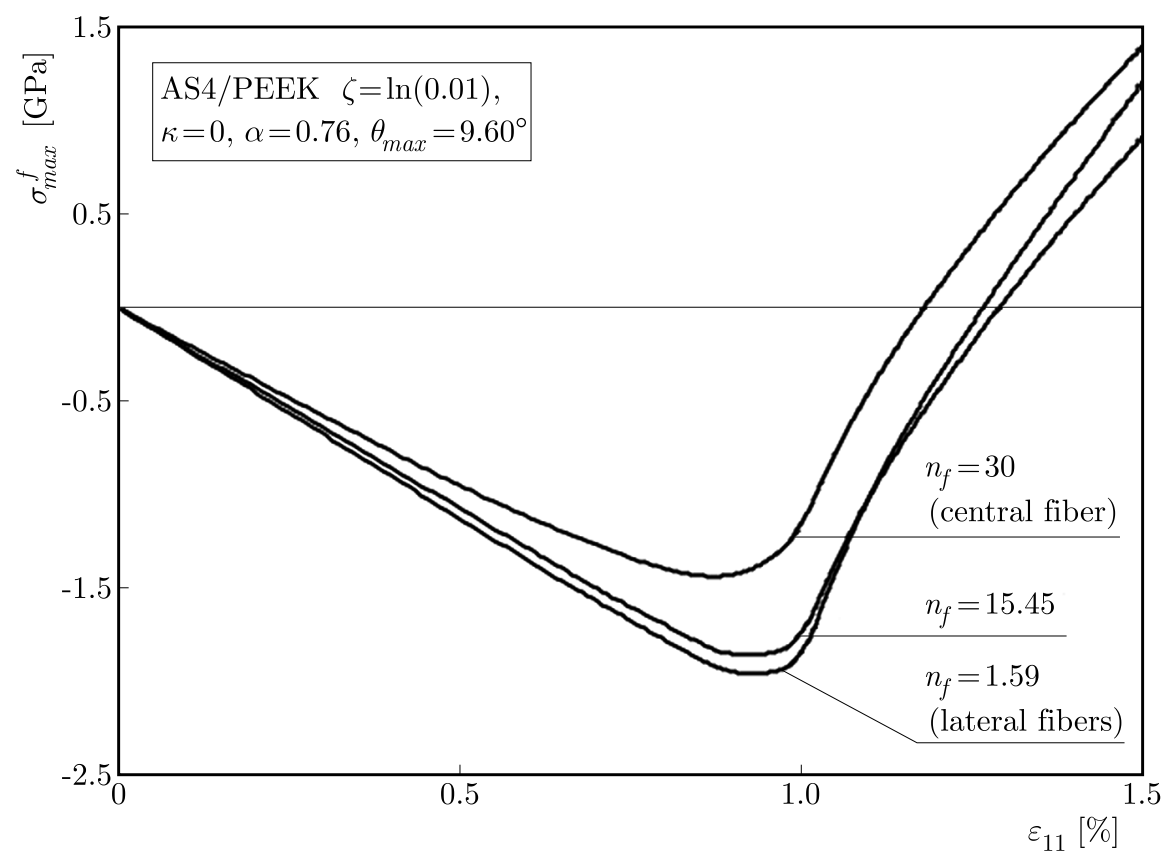

Fig. 6. Comparison of the axial stress in several fibers predicted from the model with non-uniform imperfections

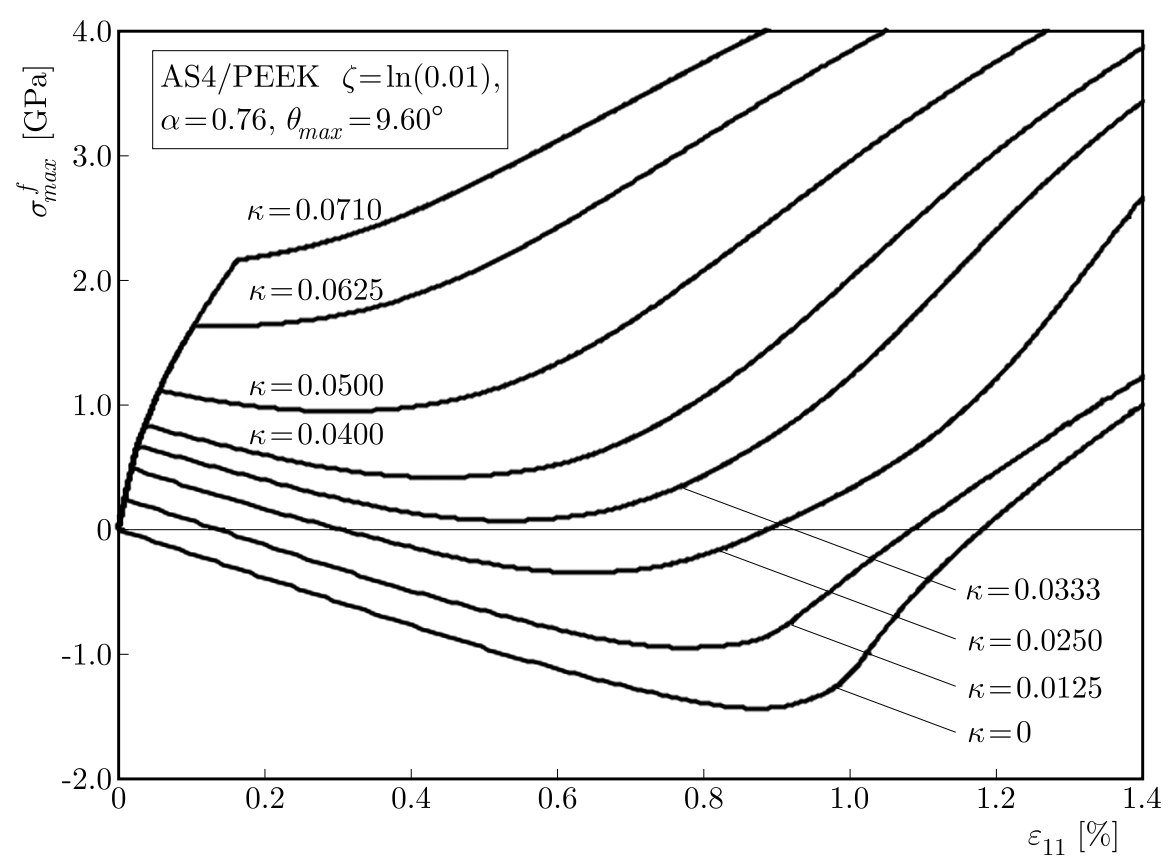

Fig. 7. Effect of the biaxial loading ratio $\kappa$ on the fiber axial stress in the AS4/PEEK composite system.

The solutions obtained from the model with non-uniform imperfections

Moreover, it is evident from these figures that the fiber axial stress can exceed the tensile strength required for fiber breaking. The tensile strength for AS4 carbon fiber is $3.45 \mathrm{GPa}$ (Kyriakides et al., 1995). It is apparent, first of all, that the breaking load of fibers decreases with increasing the applied shear loading for the two model types. It is also obvious from these figures that the load at which fibers break is lower in the case of a non-uniform distribution of imperfections. Because non-uniform models have larger initial misalignment angles than uniform ones, the tensile stress in fibers starts at lower applied strain levels in the cases with 


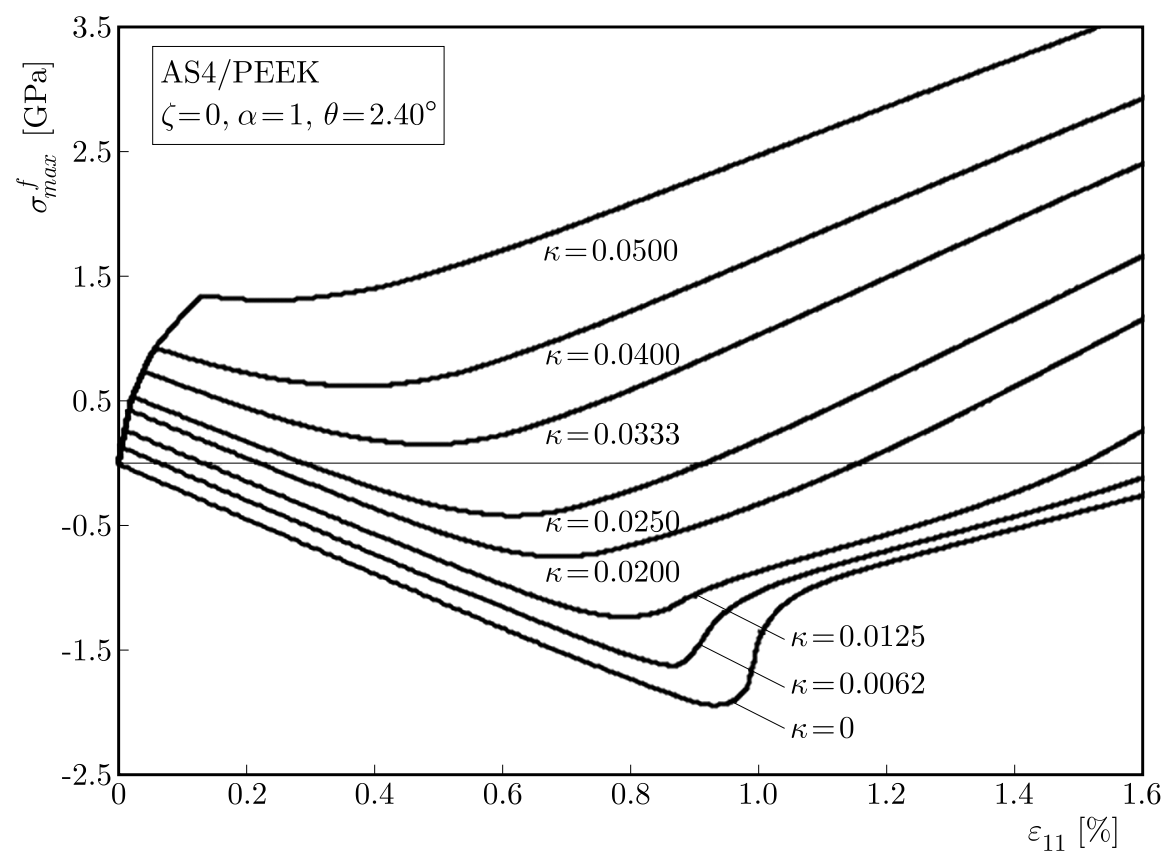

Fig. 8. Effect of the biaxial loading ratio $\kappa$ on the fiber axial stress in the AS4/PEEK composite system.

The solutions obtained from the model with uniform imperfections

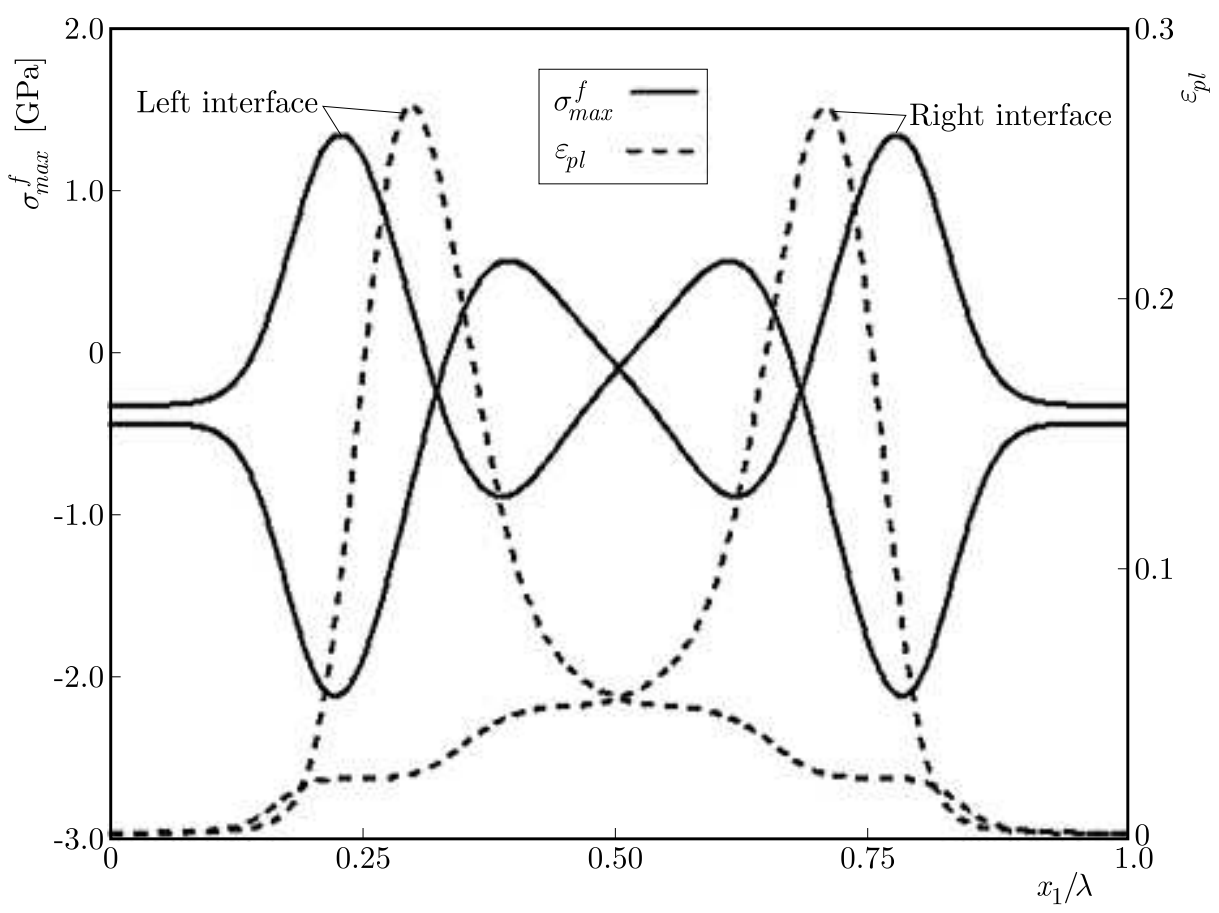

Fig. 9. Distributions of the maximum axial stress and the effective plastic strain at the interfaces between the matrix and the central fiber at the post buckling stage of deformation predicted from the model with non-uniform imperfections

non-uniform imperfections compared to the corresponding cases with uniform imperfections. As a result of this, the model with the non-uniform distribution of imperfections is better suited to deal with the fiber breaking mechanism. This means that the distribution of fiber imperfections plays a significant role both in predicting the limit load as well as in understanding failure mechanisms. 
An exemplary evaluation of kink bands in the non-uniform model under pure compressive loading is shown in Fig. 9. By comparing the locations of the maximum tensile stress with the locations of the maximum plastic strain at the interface between matrix and the central fiber, it can be seen that fibers will start breaking due to excessive bending in regions of the most intense plastic deformation. Thus, it is reasonable to suppose that the composite during compressive loading fails within kink-bands first by local matrix shearing leading to split formation between the fibers and then by fiber breaking. To summarize, on the one hand, characteristics of the localization process such as the kink-band angle and the kink-band width can be evaluated from contours of the effective plastic strain in the matrix. On the other hand, values of the maximum tensile fiber stress can be useful in identifying the ultimate failure. Experimental observations of kink band formation in the presence of shear loading (Vogler et al., 2000) show that the kink band inclinations in the PEEK matrix composite vary from $10^{\circ}-15^{\circ}$ which are somewhat lower than the values measured in similar tests under pure compression. The kink band formation under combined axial compression and in-plane shear can be evaluated from the contours of the effective plastic strain in the matrix at three stages of deformation which represent different responses of the material, namely the beginning of plastic flow, the limit load and the post-buckling behavior. The development of plastic deformation predicted from the model with the non-uniform distribution of imperfections for $\kappa=0.0125$ and 0.05 is shown in Figs. 11 and 12. For comparison purposes, the growth of the kink band across this model for the pure compression case $(\kappa=0)$ is illustrated in Fig. 10. The propagation of kinking is observed in two inclined bands that are separated from each other and clearly defined. Due to

(a)

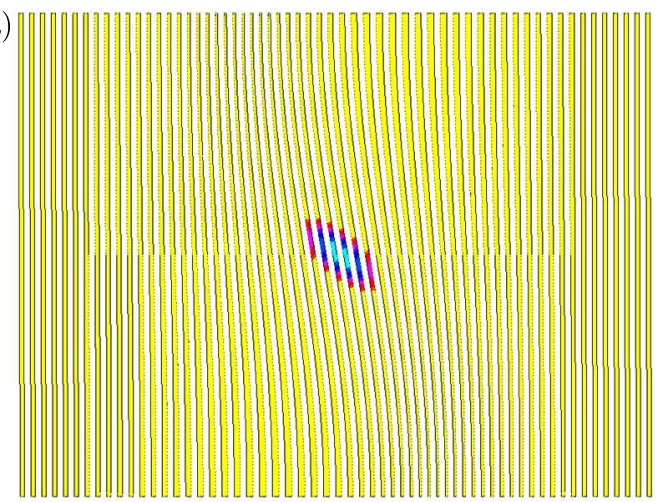

(b)

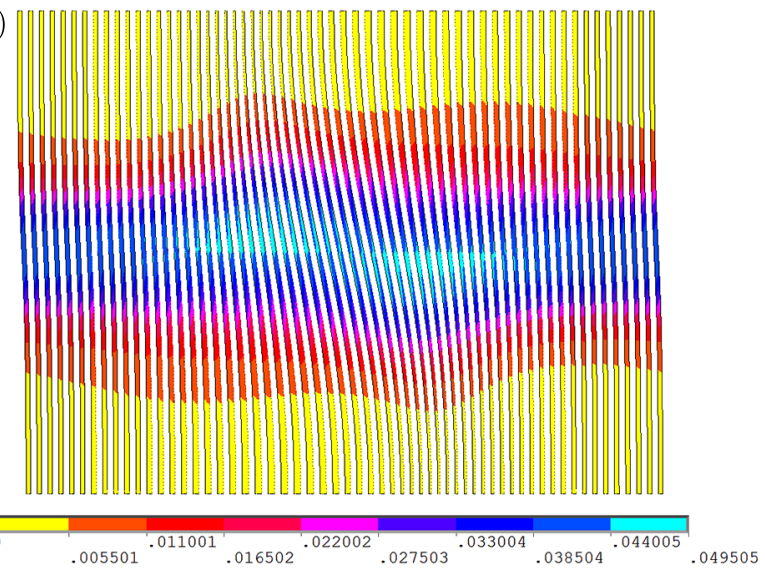

(c)
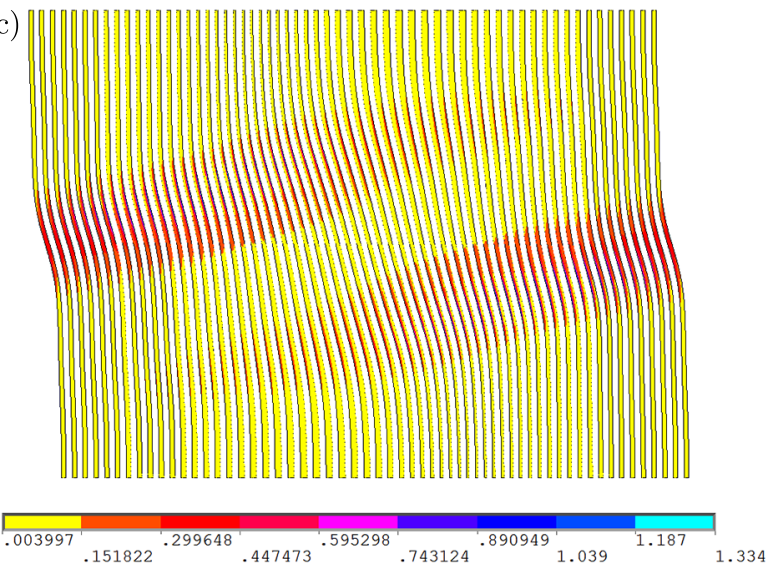

Fig. 10. Contour plots of the effective plastic strain for $\kappa=0$ at three stages of deformation corresponding to (a) the beginning of plastic flow $\left(\varepsilon_{11}=0.43 \%\right)$, (b) the limit load $\left(\varepsilon_{11}=0.99 \%\right)$,

(c) the post buckling $\left(\varepsilon_{11}=1.56 \%\right)$ 
the periodicity in the transverse direction, the solution shown in Figs. 10-12 can be regarded as a single band that spreads out from one maximum fiber misalignment to another through a region of initially straight fibers. It is easy to see that microbuckles initiate inside the models at the regions of higher misalignment. After the load begins to drop, the fibers in these models rotate. As the bending curvature increases, the bands of the most intense plastic deformation become increasingly more inclined with respect to the horizontal direction. The maximum plastic strains in these bands at the post-buckling stage are also located at the regions of higher misalignment. As it has been mentioned before (Fig. 9), the initiation of fiber breaks is expected to take place in these zones. The kink band inclinations calculated at this stage show good agreement with those measured in the experiments just before fiber breaking. It is interesting to note that models with larger values of $\kappa$ tend to have smaller inclination angles. Moreover, both the kink band width and the plastic deformation grow larger with the increasing ratio $\kappa$. This means that the region where fibers undergo significant bending is also expanding. As a result of this, the post-limit load response is less unstable for larger values of $\kappa$.
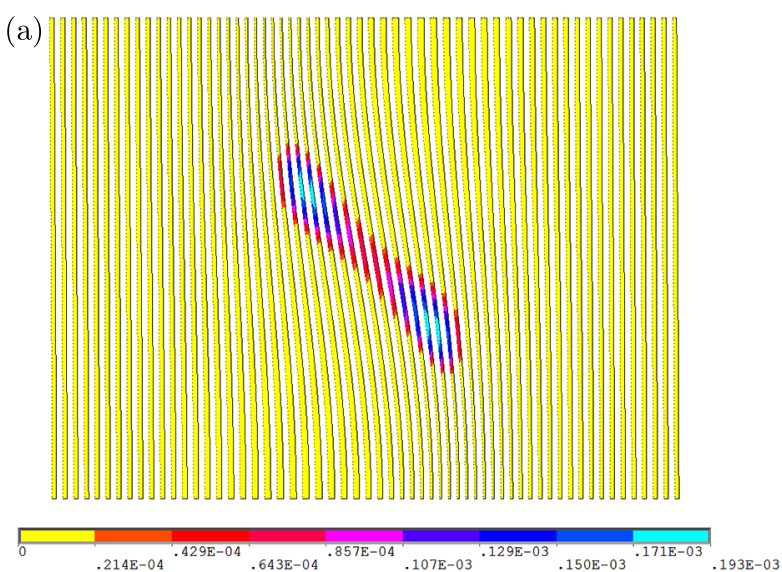

(b)

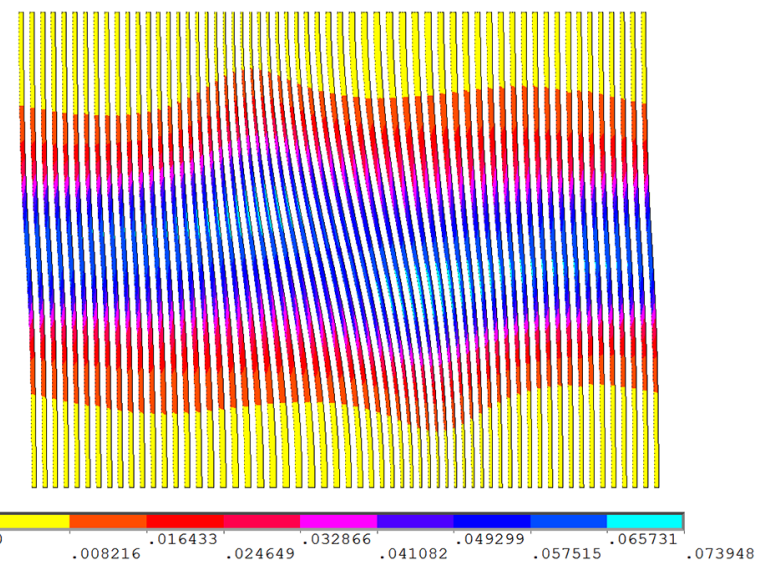

(c)

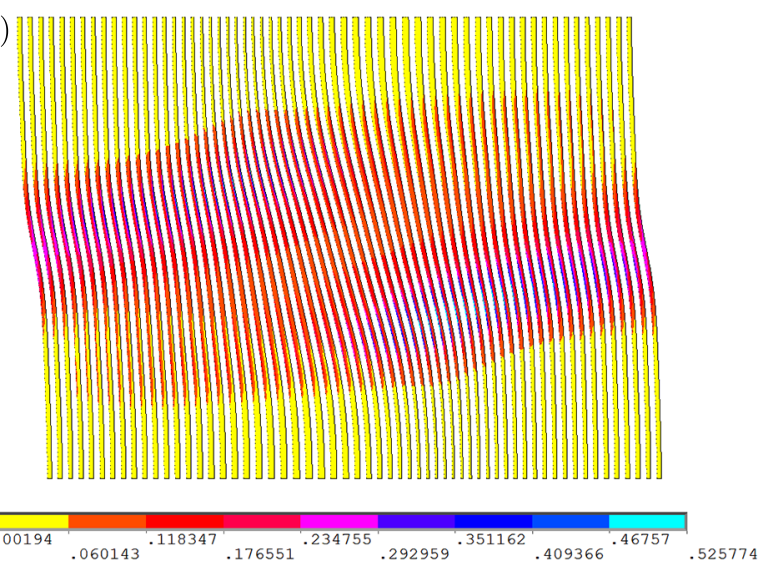

Fig. 11. Contour plots of the effective plastic strain for $\kappa=0.0125$ at three stages of deformation corresponding to (a) the beginning of plastic flow $\left(\varepsilon_{11}=0.28 \%\right)$, (b) the limit load $\left(\varepsilon_{11}=0.92 \%\right)$,

(c) the post buckling $\left(\varepsilon_{11}=1.30 \%\right)$

\subsection{Comparison with experiments and other simulations}

As it is shown in Fig. 13, the failure locus in the stress plane obtained from the non-uniform model consists of two curves that differ in mode of failure. The first curve plotted as a solid line indicates the region where the fiber buckling failure occurs. The second curve plotted as a dotted line is associated with the stable fiber bending and refers to the cases in which the axial stress in the central fiber has no minimum value. 

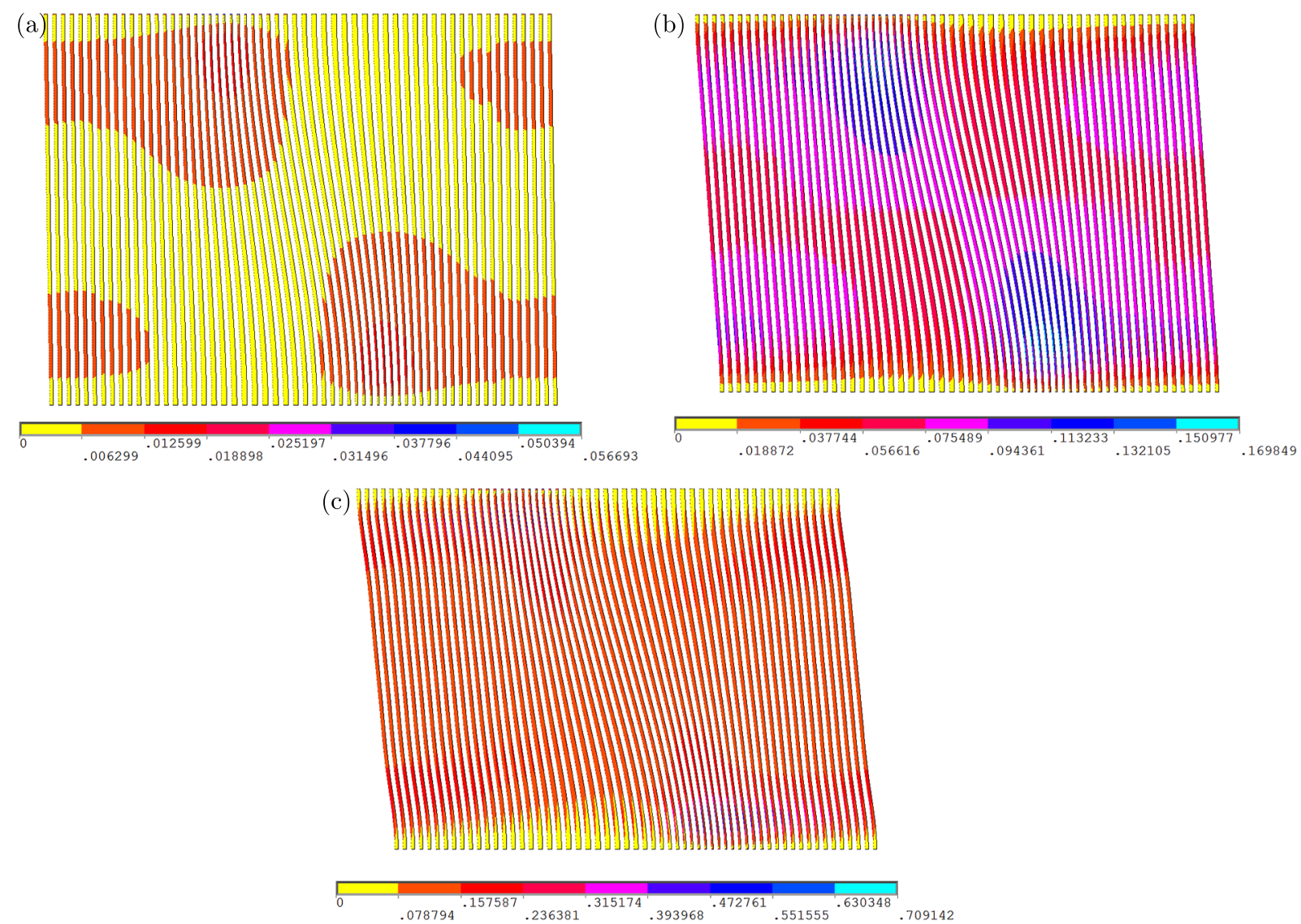

Fig. 12. Contour plots of the effective plastic strain for $\kappa=0.05$ at three stages of deformation corresponding to (a) the beginning of plastic flow $\left(\varepsilon_{11}=0.56 \%\right)$, (b) the limit load $\left(\varepsilon_{11}=0.80 \%\right)$,

(c) the post buckling $\left(\varepsilon_{11}=1.20 \%\right)$

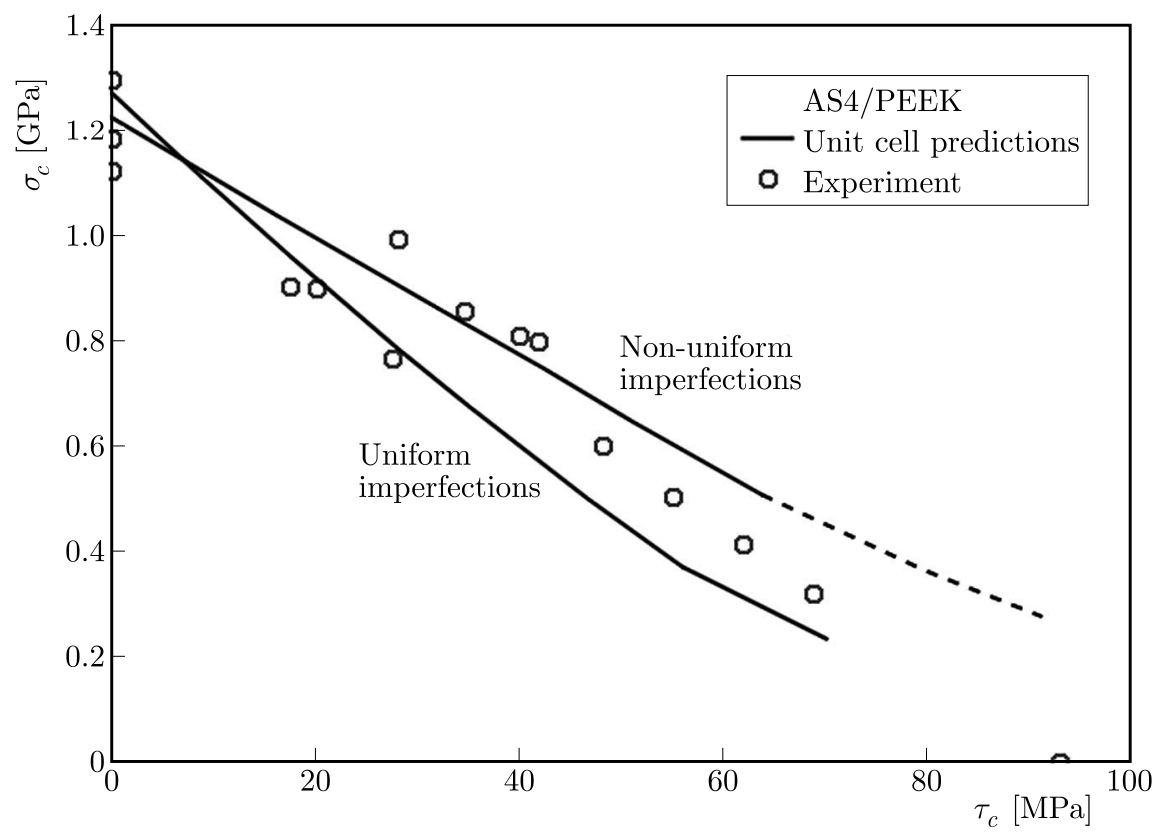

Fig. 13. Comparison of the failure loci predicted from micromechanical models with experimental results for the AS4/PEEK composite system 
The limit loads obtained from the model with non-uniform imperfections for various loading ratios are compared both with predictions based on the uniform distribution of imperfections and with experimental data available in the literature (Vogler et al., 2000). Biaxial tests for the AS4/PEEK composite have been carried out sequentially, first shear, then compression. The results of this comparison are summarized in Fig. 13. It can be seen from this figure that the compressive strength predicted from both models in the first region decreases almost linearly with increasing the applied shear loading. In the second region, the failure loci change from linear to nonlinear. It can be seen that the model with fixed amplitude imperfections shows lower biaxial strength than the model with decaying amplitude imperfections. What is surprising is that the latter case has a larger fiber misalignment angle than the first case. This finding suggests that the effect of fiber waviness on failure in shear is opposite to that in compression. This means that the waviness of the fibers in unidirectional composites promotes fiber microbuckling and inhibits matrix shearing. Although the two model types follow the trend of the experimental points, the case with non-uniform imperfections is in better agreement with the measured biaxial strength than the case with uniform imperfections. Thus, the classical approach based on the uniform distribution of imperfections underpredicts the microbuckling strength of fiber reinforced composites under combined axial compression and in-plane shear. Please note that a small calibration error for the model with uniform imperfections is visible in Fig. 13 at zero shear strength $\left(\tau_{c}=0\right)$. A better calibration of this model would move the model predictions down and, as a result of this, the effect of underestimation would be even larger.

As it has been shown in the previous Section, the models with initially wavy fibers are only destined to predict the fiber microbuckling behavior. For very large values of $\kappa$, the unidirectional composites fail by stable fiber bending. In this case, the models with initially wavy fibers overrate the failure stress because the fiber waviness acts as a barrier that blocks the in-plane shear deformation.

\section{Conclusions}

A numerical study has been performed to demonstrate that the periodic unit cell model with centrally located imperfections is able to reproduce fiber microbuckling that governs the failure of fiber reinforced composites in the case of compression combined with low and moderate shear. In particular, the proposed model better simulates the reduction in compressive strength resulting from the application of an additional shear stress than the classical model with uniform imperfections. Moreover, the kink-band inclination calculated from the proposed model at the post-buckling stage is in the same range as that measured in experiments. It has also been found that the buckling instability is inhibited by the presence of high tensile stress in fibers arising from the shear loading. The simulations show that when the applied shear stress is sufficiently large, the fiber microbuckling mechanism lapses and unidirectional composites fail by stable fiber bending.

Acknowledgment

The financial support of the National Science Centre of Poland under contract DEC$-2011 / 03 / \mathrm{D} / \mathrm{ST} 8 / 04817$ is thankfully acknowledged.

\section{References}

1. ANSYS, 2016, Mechanical APDL theory reference, release 17.1, Cannonsburg PA

2. Barulich N.D., Godoy L.A., Barbero E.J., 2016, On micro-buckling of unidirectional fiber-reinforced composites by means of computational micromechanics, Latin American Journal of Solids and Structures, 13, 3085-3106 
3. Byskov E., Christoffersen J., Christensen C.D., Poulsen J.S., 2002, Kinkband formation in wood and fiber composites-morphology and analysis, International Journal of Solids and Structures, 39, 3649-3673

4. Crisfield M.A., 1981, A fast and incremental/iterative solution procedure that handles snap-through, Computers and Structures, 13, 55-62

5. Czabaj M.W., Riccio M.L., Whitacre W.W., 2014, Numerical reconstruction of graphite/epoxy composite microstructure based on sub-micron resolution X-ray computed tomography, Composites Science and Technology, 105, 174-182

6. Drucker D.C., Prager W., 1952, Soil mechanics and plastic analysis for limit design, Quarterly of Applied Mathematics, 10, 157-165

7. Gregory J.R., Spearing S.M., 2005, Nanoindentation of neat and in situ polymers in polymer-matrix composites, Composites Science and Technology, 65, 595-607

8. Gutkin R., Pinho S.T., Robinson P., Curtis P.T., 2010a, Micro-mechanical modeling of shear-driven fibre compressive failure and of fibre kinking for failure envelope generation in CFRP laminates, Composites Science and Technology, 70, 1214-1222

9. Gutkin R., Pinho S.T., Robinson P., Curtis P.T., 2010b, On the transition from shear-driven fibre compressive failure to fibre kinking in notched CFRP laminates under longitudinal compression, Composites Science and Technology, 70, 1223-1231

10. Gutkin R., Pinho S.T., Robinson P., Curtis P.T., 2011, A finite fracture mechanics formulation to predict fibre kinking and splitting in CFRP under combined longitudinal compression and in-plane shear, Mechanics of Materials, 43, 730-739

11. Guynn E.G., Ochoa O.O., Bradley W.L., 1992, A parametric study of variables that affect fiber microbuckling initiation in composite laminates. Part I - Analyses, Journal of Composite Materials, 26, 1594-1611

12. Hsu S.Y., Vogler T.J., Kyriakides S., 1998, Compressive strength predictions for fiber composites, Journal of Applied Mechanics, 65, 7-16

13. Hsu S.Y., Vogler T.J., Kyriakides S., 1999, On the axial propagation of kink bands in fiber composites. Part II - Analysis, International Journal of Solids and Structures, 36, 575-595

14. Jelf P.M., Fleck N.A., 1994, The failure of composite tubes due to combined compression and torsion, Journal of Materials Science, 29, 3080-3084

15. Kinloch A.J., Young R.J., 1983, Fracture Behavior of Polymers, Elsevier

16. Kyriakides S., Arseculeratne R., Perry E.J., Liechti K.M., 1995, On the compressive failure of fiber reinforced composites, International Journal of Solids and Structures, 32, 689-738

17. Kyriakides S., Ruff A.E., 1997, Aspects of the failure and postfailure of fiber composites in compression, Journal of Composite Materials, 31, 2000-2037

18. Morais A.B., 1996, Modelling lamina longitudinal compression strength of carbon fibre composite laminates, Journal of Composite Materials, 30, 1115-1131

19. Naya F., Herraez M., Lopes C.S., Gonzalez C., van Der Veen S., Pons F., 2017, Computational micromechanics of fiber kinking in unidirectional FRP under different environmental conditions, Composites Science and Technology, 144, 26-35

20. Pansart S., Sinapius M., Gabbert U., 2009, A comprehensive explanation of compression strength differences between various CFRP materials: micro-meso model, predictions, parameter studies, Composites Part A, 40, 376-387

21. Pimenta S., Gutkin R., Pinho S.T., Robinson P., 2009, A micromechanical model for kink-band formation. Part I: Experimental study and numerical modeling, Composites Science and Technology, 69, 948-955

22. PrabhakAR P., WAAS A.M., 2013, Interaction between kinking and splitting in the compressive failure of unidirectional fiber reinforced laminated composites, Composite Structures, 98, 85-92 
23. Quinson R., Perez J., Rink M., Pavan A., 1997, Yield criteria for amorphous glassy polymers, Journal of Materials Science, 32, 1371-1379

24. Romanowicz M., 2014, Initiation of kink bands from regions of higher misalignment in carbon fiber reinforced polymers. Journal of Composite Materials, 48, 2387-2399

25. Vogler T.J., Hsu S.Y., Kyriakides S., 2000, Composite failure under combined compression and shear, International Journal of Solids and Structures, 37, 1765-1791

26. Vogler T.J., Hsu S.Y., Kyriakides S., 2001, On the initiation and growth of kink bands in fiber composites, Part II - Analysis, International Journal of Solids and Structures, 38, 2653-2682

27. Wilhelmsson D., Gutkin R., Edgren F., Asp L.E., 2018, An experimental study of fibre waviness and its effects on compressive properties of unidirectional NCF composites, Composites Part A, 107, 665-674

Manuscript received August 18, 2017; accepted for print September 20, 2018 